\title{
Input-Output Linearization with Simultaneous Decoupling by Restricted State Feedback
}

\author{
K.G.ARVANITIS ${ }^{1,2}$, A.S.TSIRIKOS ${ }^{1,2}$ and N.E.MASTORAKIS ${ }^{2}$ \\ ${ }^{1}$ )NATIONAL TECHNICAL UNIVERSITY OF ATHENS \\ DIVISION OF COMPUTER SCIENCE \\ DEPARTMENT OF ELECTRICAL AND COMPUTER ENGINEERING \\ 15773 ZOGRAPHOU, ATHENS, GREECE \\ $\left({ }^{1,2}\right)$ MILITARY INSTITUTIONS OF UNIVERSITY EDUCATION \\ HELLENIC NAVAL ACADEMY \\ CHAIR OF COMPUTER SCIENCE \\ 18539, HATZIKYRIAKOY, PIRAEUS, GREECE
}

\begin{abstract}
The input-output (i/o) linearization with simultaneous decoupling problem of nonsquare nonlinear systems via restricted state feedback is investigated. The problem is treated on the basis of a new algebraic approach, whose main feature is that it reduces the determination of the admissible state feedback control laws to the solution of an algebraic and a first order partial differential systems of equations.Verifiable necessary and sufficient conditions, of algebraic nature, for the solvability of the aforementioned problem, which rely on the solvability of these systems equations, are established. Moreover, an explicit expression for a special admissible restricted state feedback controller is analytically derived.
\end{abstract}

\section{Introduction}

State feedback diagonal decoupling problem has long been the focus of attention by many control designers, since it offers a new dimension of flexibility to the design process. In the case where nonsingular input transformation is assumed, the problem has been extensively treated in the past (see e.g. [1],[2],[5] for linear and [3]-[5],[17] for nonlinear systems).

Without any assumption on the input transformation, the diagonal decoupling, called in this case the Morgan's problem [6], was studied in the past in [7]-[11], for linear time invariant systems and in [12] for nonlinear systems.Up to now, only special cases of the Morgan's problem, in which restrictive assumptions are added to the system, were treated (see [11], [12] for an extended analysis of this issue). For these particular cases, necessary and/or sufficient conditions for the solvability of the problem were established [7], [9], [10], [12]. Nevertheless, the general static Morgan's problem is still open. An alternative way to approach Morgan's problem, is the one based on restricted state feedback. When restricted state feedback is applied, general results can be obtained for linear systems [13]-[15] as well as for nonlinear systems [16]. In particular, for the case of nonlinear systems, the problem of i/o linearization and decoupling, treated in this paper, is investigated also in [16], wherein a geometric approach is used to establish necessary and sufficient conditions for the solvability of the problem. In the approach reported in [16], a priori assumption on the relative degrees of the $\mathrm{i} / \mathrm{o}$ linearized and decoupled closed-loop system is made. Furthermore, 
the solvability criteria suggested there, are of geometric nature, which intuitively may be relaxed to verifiable ones.

In the present paper, a new approach to the i/o linearization with simultaneous decoupling problem of nonsquare nonlinear systems, using restricted state feedback is presented. The proposed approach is motivated by the results reported in [15] and it is a purely algebraic one. Its main feature consists in that it reduces the determination of the admissible controller to the solution of a system of algebraic equations and of a system of first order partial differential equations. On the basis of these systems of equations, easily verifiable necessary and sufficient conditions of simple algebraic nature are established for the solvability of the aforementioned problem. It is emphasised also that these conditions constitute sufficient conditions for the static Morgan's problem of nonlinear systems. Furthermore, our approach directly offers an explicit expression for a special admissible restricted static state feedback controller. Note also that, the present approach provides the fundamental equations needed for the characterisation of all admissible controllers as well as of the respective i/o linearized and simultaneously decoupled closed-loop responses. The proposed technique gives some new insights to the decoupling problem of nonlinear systems, since it elaborates the problem in a more general context in comparison with known results.

\section{Preliminaries and problem statement}

Consider the nonlinear nonsquare analytic system described in state - space by

$$
\dot{\mathbf{x}}=\mathbf{g}_{\mathbf{0}}(\mathbf{x})+\mathbf{G}(\mathbf{x}) \mathbf{u}, \mathbf{x}(\mathbf{0})=\mathbf{x}_{\mathbf{0}}, \mathbf{y}=\mathbf{h}(\mathbf{x})
$$

where the input vector $\mathbf{u} \in R^{m}$ is a vector of analytic functions, the output vector $\mathbf{y} \in \mathbf{R}^{\mathbf{p}}$ and the state $\mathbf{x}$ belongs to an open subset $X$ of $R^{n}$. In (2.1), $\mathbf{g}_{\mathbf{0}}$ and $\mathbf{G}$ are analytic matrix valued functions of $\mathbf{x}$ and $\mathbf{h}(\mathbf{x})$ is an analytic mapping from $X$ to $R^{p}$.

Furthermore, consider the nonlinear restricted state feedback control law

$$
\mathbf{u}=\mathbf{B}(\mathbf{x})(\mathbf{a}(\mathbf{x})+\mathbf{w})
$$

where the external input $\mathbf{w} \in R^{p}$ is a vector of analytic functions, and $\mathbf{a}(\mathbf{x})$ and $\mathbf{B}(\mathbf{x})$ are analytic matrix valued functions of $\mathbf{x}$.

Consider now applying to system (2.1) the control law (2.2). The resulting closedloop system will have the form

$$
\dot{\mathbf{x}}=\overline{\mathbf{g}}_{0}(\mathbf{x})+\overline{\mathbf{G}}(\mathbf{x}) \mathbf{w}, \mathbf{x}(\mathbf{0})=\mathbf{x}_{\mathbf{0}}, \mathbf{y}=\mathbf{h}(\mathbf{x})
$$

where

$$
\overline{\mathbf{g}}_{0}(\mathbf{x})=\mathbf{g}_{\mathbf{0}}(\mathbf{x})+\mathbf{G}(\mathbf{x}) \mathbf{B}(\mathbf{x}) \mathbf{a}(\mathbf{x}), \overline{\mathbf{G}}(\mathbf{x})=\mathbf{G}(\mathbf{x}) \mathbf{B}(\mathbf{x})
$$

Observe that the closed-loop system (2.3a) is square with $\mathrm{p}$ number of inputs and outputs. Hence, it is reasonable to investigate whether this system is simultaneously i/o linearized and decoupled.

In what follows we denote by $J_{\rho}$ the set of integers $\{1,2, \ldots, \rho\}$, where $\rho \in Z^{+}$, and by $J_{\infty}$ the set of positive integers $\{1, \ldots, \infty\}$. The following two Lemmas provide us criteria for system (2.3) to be i/o linearized or decoupled (see [17] for details].

\section{Lemma 2.1.}

The nonlinear system (2.3) is i/o linearized if the following relations hold

$$
\underset{g_{j}}{L_{-}} L_{g_{0}}^{k} h_{i}(x)=\delta_{i, j, k} \forall i, j \in J_{p} \text { and } k \in\{0\} \cup J_{2 n-1}
$$


in the neighbourhood of $\mathbf{x}_{\mathbf{0}}$, where $\overline{\mathbf{g}}_{i}(x), i \in J_{m}$, denotes the i-th column of $\overline{\mathbf{G}}(\mathbf{x}), \boldsymbol{\delta}_{i, j, k}, i \in J_{p}$ and $k \in\{0\} \cup J_{2 n-1}$, are real numbers and $h_{i}(\mathbf{x}), i \in J_{p}$, denotes the ith component of $\mathbf{h}(\mathbf{x})$.

\section{Lemma 2.2.}

The nonlinear system (2.3) is decoupled if the following relations simultaneously hold

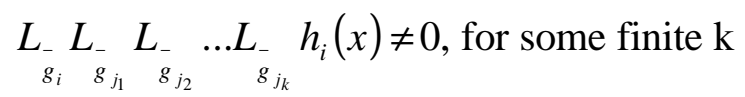

$$
\begin{aligned}
& L_{g_{j}} L_{g_{j_{1}}} L_{g_{j_{2}}} L_{-} \ldots L_{g_{j_{k}}} h_{i}(x)=0 \\
& \forall i, j \in J_{p}, j \neq i, j_{1}, \ldots, j_{k} \in\{0, i\} \text { and } k \in\{0\} \cup J_{\infty}
\end{aligned}
$$

Combining appropriately Lemmas 2.1 and 2.2 it is obvious that a criterion for system (2.3) to be simultaneously $\mathrm{i} / \mathrm{o}$ linearized and decoupled is given by the following Theorem.

\section{Theorem 2.1.}

The nonlinear system (2.3) is i/o linearized and simultaneously decoupled if the following relations simultaneously hold

$$
\begin{gathered}
L_{-} L_{g_{i}}^{k} h_{g_{0}}(x)=\delta_{k, i} \forall i \in J_{p} \text { and } k \in\{0\} \cup J_{2 n-1} \\
L_{-} L_{g_{j}} L_{g_{0}}^{k} h_{i}(x)=0, \forall i, j \in J_{p}, j \neq i, k \in\{0\} \cup J_{2 n-1}
\end{gathered}
$$

in the neighbourhood of $\mathbf{x}_{\mathbf{0}}$, and $\delta_{k, i}$, for $i \in J_{m}$ and $k \in\{0\} \cup J_{2 n-1}$, are real numbers not all zero.

On the basis of Theorem 2.1 the problem of $\mathrm{i} / \mathrm{o}$ linearization and simultaneous decoupling for system (2.1) can be stated as follows:

\section{Definition 2.1.}

The combined problem of i/o linearization with decoupling for the nonsquare system (2.1) via restricted state feedback consists in determining a control law of the form (2.2) such that the closed-loop system (2.3) is i/o linearized and simultaneously decoupled, i.e. relations ( $2.4 \mathrm{a}$ and $\mathrm{b}$ ) of Theorem 2.1 , are simultaneously satisfied.

The approach presented in this paper in order to treat the problem of i/o linearization and decoupling rely on the Definition 2.1. To simplify the presentation of our approach we give at this point some useful material.

\section{Definition 2.2.}

The characteristic numbers of the nonlinear system (2.1) are the nonnegative integers $\gamma_{i} s$ for $i \in J_{p}$, satisfying the following relations

$\forall x$ around $\mathbf{x}_{\mathbf{0}}$.

$$
\begin{aligned}
& \underset{g_{j}}{L_{g_{j}}} L_{g_{0}}^{k} h_{i}(x)=0, \forall j \in J_{m} \text { and } \mathrm{k}<\gamma_{i} \\
& \underset{g_{j}}{L_{g_{0}}} L_{g_{0}}^{\gamma_{i}} h_{i}(x) \neq 0 \text {, for some } j \in J_{m}
\end{aligned}
$$

It is remarked that the characteristic numbers $\gamma_{i}{ }^{\prime} s$ of (2.1) depend on the unknown matrix valued function $\mathbf{B}(\mathbf{x})$ of the control law. 
The following Lemma states some properties of the characteristic numbers which may be easily proven and will be useful in the sequel.

\section{Theorem 2.2}

The nonlinear system (2.1) may be i/o linearized and simultaneously decoupled under the restricted state feedback (2.2) if there exist matrix valued functions $\mathbf{a}(\mathbf{x})$ and $\mathbf{B}(\mathbf{x})$ such that the following relations simultaneously hold

$$
\begin{gathered}
L_{-} L_{g_{i}}^{\gamma_{g_{0}}+k} h_{i}(x)=\mu_{k, i}, \\
L_{-} L_{g_{j}}^{\gamma_{g_{0}}+k} h_{i}(x)=0, \forall j \in J_{m}, j \neq i
\end{gathered}
$$

where $\mu_{k, i} \stackrel{\Delta}{=} \delta_{d_{i}+k, i}$, within at least one $\mu_{k, i} \neq 0, \forall i \in J_{p}$ and $k \in\{0\} \cup J_{2 n-1}$.

Theorem 2.2 gives a mathematical formulation of the i/o linearization with simultaneous decoupling problem for nonsquare nonlinear systems by restricted static state feedback. Relations $(2.5 \mathrm{a}, \mathrm{b})$ will be the basis of the analysis of the problem that follows.

\section{Derivation of the design equations}

In what follows, our aim is to manipulate relations $(2.5 \mathrm{a}, \mathrm{b})$ in order to obtain a more appropriate relation for the study of the i/o linearization and decoupling problem via restricted state feedback. Keeping this in mind, the following Lemma can be proven using the perfect inductive method [17].

\section{Lemma 3.1.}

The following equality holds for any $k \in\{0\} \cup J_{\infty}$

$$
\begin{array}{r}
L_{g_{0}}^{\gamma_{i}+k} h_{i}(x)=L_{g_{0}}^{\gamma_{i}+k} h_{i}(x) \\
+\sum_{j=1}^{\gamma_{i}+k} L_{g_{0}}^{\gamma_{i}+k-j}\left[\left(L_{-} L_{-}^{j-1} h_{i}(x)\right)(\mathbf{a}(\mathbf{x}))\right]
\end{array}
$$

On the basis of Lemma 3.1, relations (2.5) may be rewritten in the following form

$$
\begin{gathered}
L_{G}\left(L_{g_{0}}{ }^{\gamma_{i}} h_{i}(x)\right)=\mu_{0, i} e_{i}^{T} \\
L_{G}\left[\sum_{j=1}^{\gamma_{i}+k} L_{g_{0}}^{\gamma_{i}+k-j}\left[\left(L_{-} L_{-}^{j-1} h_{i}(x)\right)(\mathbf{a}(\mathbf{x}))\right]\right]+L_{-}\left(L_{g_{0}}^{\gamma_{i}+k} h_{i}(x)\right)=\mu_{k, i} e_{i}^{T}
\end{gathered}
$$

$\forall i \in J_{p}$ and $k \in\{0\} \cup J_{2 n}$ where use was made of the definition of the characteristic numbers $\gamma_{i} s$. Furthermore, substituting appropriately (2.5) into (3.2) we obtain

$$
\begin{gathered}
L_{G}\left(L_{g_{0}}^{\gamma_{i}} h_{i}(x)\right)=\mu_{0, i} e_{i}^{T} \\
L_{G}\left(L_{g_{0}}^{\gamma_{i}+k} h_{i}(x)\right)+\sum_{j=0}^{k-1} \mu_{j, i} L_{G} L_{g_{0}}^{k-j-1} \mathbf{a}_{i}(\mathbf{x})=\mu_{k, i} e_{i}^{T}
\end{gathered}
$$

$\forall i \in J_{p}$ and $k \in J_{2 n}$ where $e_{i}^{T}$ denotes the i-th row of the mxm identity matrix $a_{i}(x)$ denotes the $\mathrm{i}$-th element of the vector valued function $\mathbf{a}(\mathbf{x})$. To produce (3.3b) use was made of the definition of the characteristic numbers and of Lemma 2.1. We mention that from (3.3a) and the definition of the characteristic numbers we readily conclude that $\mu_{0, i} \neq 0$, for $i \in J_{p}$. 
To facilitate the solution of (3.3) with respect to $a_{i}(x), \mathbf{B}(\mathbf{x})$ and $\mu_{k, i}, i \in J_{p}$ and $k \in\{0\} \cup J_{2 n}$, observe that the decoupled and i/o linearized closed-loop system has an i/o description of the form

$$
\mathrm{H}(\mathrm{s})=\underset{i \in J_{p}}{\operatorname{diag}}\left\{h_{i}(s)\right\}
$$

where $h_{i}(s)$ have the form

$$
h_{i}(s)=s^{-\left(\gamma_{i}+1\right)} \sum_{k \in J_{\infty}} \mu_{k, i} s^{-k}
$$

$i \in J_{p}$, where $\mu_{0, i} \neq 0$. To produce (3.5) use was made of the definition of the characteristic numbers and of Lemma 2.3. We next denote by $\hat{H}(s)$ the following transfer function

$$
\hat{H}(s)=\underset{i \in J_{p}}{\operatorname{diag}}\left\{\hat{h}_{i}(s)\right\} \stackrel{\Delta}{=} H^{-1}(s), \hat{h}_{i}(s)=h_{i}^{-1}(s) .
$$

It is not difficult to see that $\hat{h}_{i}(s), i \in J_{p}$, have the form

$$
\hat{h}_{i}(s)=s^{\gamma_{i}+1} \sum_{k \in J_{\infty}} \rho_{k, i} s^{-k}
$$

where use was made of the fact that $\mu_{0, i} \neq 0, i \in J_{p}$. The Markov parameters $\mu_{k, i}$, $k \in\{0\} \cup J_{2 n}$ and $i \in J_{p}$, may be given as a function of $\rho_{k, i}, k \in\{0\} \cup J_{2 n}$ and $i \in J_{p}$, as

$$
\begin{gathered}
\mu_{0, i}=\rho_{0, i}^{-1} \\
\mu_{k, i}=-\rho_{0, i}^{-1} \sum_{j=0}^{k-1} \mu_{k-j, i} \rho_{j, i}
\end{gathered}
$$

for $i \in J_{p}$ and $k \in J_{2 n}$. Introducing (3.6) into (3.3) and solving the resulting equations with respect to $L_{G} L_{g_{0}}^{\gamma_{i}} h_{i}(x)$ and $L_{G} L_{g_{0}}^{k} a_{i}(x)$, we obtain

$$
\begin{gathered}
L_{G} L_{g_{0}}^{\gamma_{i}} h_{i}(x)-\rho_{0, i}^{-1} e_{i}^{T}=0 \\
L_{G} L_{g_{0}}^{k}\left(a_{i}(x)\right)+\sum_{j=0}^{k+1} \rho_{j, i} L_{G} L_{g_{0}}^{k-j+\gamma_{i}+1}\left(h_{i}(x)\right)=0
\end{gathered}
$$

for $i \in J_{p}$ and $k \in J_{2 n}$. Equation (3.7a) is linear algebraic with respect to $\overline{\mathbf{G}}(\mathbf{x})$ while (3.7b) is a system of partial differential equations with respect to $a_{i}(x)$. Note that for such a system of partial differential equations one cannot, in general guarantee the existence of a solution. However, due to the specific form of (3.7b), it is plausible to establish conditions for its solvability. To this end, we state the following Lemma, which can be easily proven by induction (see [15], [17] for a similar result].

\section{Lemma 3.2.}

For $k \in J_{2 n}$, the following identity holds

$$
L_{G} L_{g_{0}}^{k} a_{i}(x)=(-1)^{k} d\left(a_{i}(x)\right) a d_{g_{0}}^{k} \overline{\mathbf{G}}(x)+\sum_{j=o}^{k-1}(-1)^{j+k}\left(\begin{array}{l}
k \\
j
\end{array}\right) L_{g_{0}}^{k-j}\left[L_{G} L_{g_{0}}^{k} a_{i}(x)\right]
$$

where $\operatorname{ad}_{g_{0}}^{k} \overline{\mathbf{G}}(x)$ denotes the iterated Lie bracket operation defined by 


$$
\begin{gathered}
a d_{g_{0}}^{k} \overline{\mathbf{G}}(x)=\left[a d_{g_{0}}^{k} \bar{g}_{1}(x) \vdots a d_{g_{0}}^{k} g_{2}(x) \vdots \ldots \vdots a d_{g_{0}}^{k} \bar{g}_{m}(x)\right], \\
a d_{g_{0}}^{\lambda} g_{\rho}^{-}(x)=a d_{g_{0}}^{\lambda-1} g_{\rho}^{-}(x) \text { for } \lambda=2,3, \ldots .
\end{gathered}
$$

and where

$$
\begin{gathered}
a d_{g_{0}} g_{\rho}(x)=\frac{a g_{\rho}^{-}(x)}{a(x)} g_{0}(x)-\frac{a g_{0}(x)}{a(x)} g_{\rho}^{-}(x) \\
a d_{g_{0}}^{0} g_{\rho}^{-}(x)=g_{\rho}(x)
\end{gathered}
$$

On the basis of Lemma 3.2, equations (3.7) may be rewritten in the following form

$$
\begin{gathered}
e_{i}^{T}-\rho_{0, i} L_{G} L_{g_{0}}^{\gamma_{i}} h_{i}(x)=0 \\
L_{G} a_{i}(x)+\rho_{0, i} L_{G} L_{g_{0}}^{\gamma_{i}+1} h_{i}(x)+\rho_{1, i} L_{G} L_{g_{0}}^{\gamma_{i}+1} h_{i}(x)=0 \\
(-1)^{k} d\left(a_{i}(x)\right) a d_{g_{0}}^{k} \overline{\mathbf{G}}(\mathbf{x})+ \\
\sum_{j=0}^{k-1}(-1)^{j+k}\left(\begin{array}{c}
k \\
j
\end{array}\right) L_{g_{0}}^{k-j}\left[L_{G} L_{g_{0}}^{j} h_{i}(x)\right]+ \\
\sum_{j=0}^{k+1} \rho_{0, i}(-1)^{k-j+\gamma_{i}+1} d\left(h_{i}(x)\right) a d_{g_{0}}^{k-j+\gamma_{i}+1} \overline{\mathbf{G}}(x)+ \\
\sum_{\lambda=0}^{k+1} \rho_{\lambda, i} \sum_{j=0}^{k-\lambda+\gamma}(-1)^{j+k-\lambda+\gamma_{i}+1}\left(k-\lambda+d_{i}+1\right) \\
x L_{g_{0}}^{k-\lambda+\gamma_{i}+1-j}\left[L_{G} L_{g_{0}}^{j} h(x)\right]=0
\end{gathered}
$$

$k \in J_{2 n}$. Using the definition of Lie bracket operation, equations (3.8) take on the form

$$
\begin{gathered}
e_{i}^{T}-\rho_{0, i} L_{G} L_{g_{0}}^{\gamma_{i}} h_{i}(x)=0 \\
d\left(a_{i}(x)\right) \overline{\mathbf{G}}(\mathbf{x})+\rho_{0, i} d\left(L_{g_{0}}^{\gamma_{i}+1} h_{i}(x)\right) \overline{\mathbf{G}}(\mathbf{x})+\rho_{1, i} d\left(L_{g_{0}}^{\gamma_{i}+1} h_{i}(x)\right) \overline{\mathbf{G}}(\mathbf{x})=0 \\
d\left(a_{i}(x)\right) a d_{g_{0}}^{k} \overline{\mathbf{G}}(\mathbf{x})+\rho_{0, i} d\left(L_{g_{0}}^{\gamma_{i}+1} h_{i}(x)\right) a d_{g_{0}}^{k} \overline{\mathbf{G}}(\mathbf{x})+\rho_{1, i} d\left(L_{g_{0}}^{\gamma_{i}} h_{i}(x)\right) a d_{g_{0}}^{k} \overline{\mathbf{G}}(\mathbf{x})+ \\
\sum_{\lambda=0}^{k-1}(-1)^{\lambda+1} \rho_{\lambda+2, i} \sum_{j=0}^{k-\lambda-1}\left(\begin{array}{c}
\lambda+j \\
\lambda
\end{array}\right) L_{g_{0}}^{j}\left[d\left(L_{g_{0}}^{\gamma_{i}} h_{i}(x)\right) a d_{g_{0}}^{k-\lambda-j-1} \overline{\mathbf{G}}(\mathbf{x})\right]=0
\end{gathered}
$$

$k \in J_{2 n}$. It is mentioned that $(3.9 \mathrm{~b}, \mathrm{c})$ is a system of first order partial differential equations with respect to $a_{i}(x)$, whose solvability criteria are well established.

The following Theorem can now be formulated.

\section{Theorem 3.1.}

The i/o linearization with simultaneous decoupling problem by the restricted state feedback (2.2), is equivalent to the solvability of the following equations with unknowns $a_{i}(x), \mathbf{B}(\mathbf{x})$ and $\rho_{k, i}, k \in\{0\} \cup J_{2 n}, i \in J_{p}$

$$
\begin{gathered}
\rho_{0, i} L_{G} L_{g_{0}}^{\gamma_{i}} h_{i}(x)=e_{i}^{T} \\
\theta_{\mathbf{i}}(\mathbf{x}) \Pi_{\mathbf{i}}(\mathbf{x})=\mathbf{0}
\end{gathered}
$$

where 


$$
\begin{gathered}
\theta_{i}(x)=\left\lfloor d\left(a_{i}(x)\right): \rho_{0, i} \vdots \rho_{1, i}:-\rho_{2, i} \vdots \ldots . .(-1)^{2 n-1} \rho_{2 n, i}\right\rfloor \\
\Pi_{i}(x)=\left[\Pi_{i}^{a}(x): \Pi_{i}^{b}(x)\right]
\end{gathered}
$$

and where

$$
\begin{aligned}
& \Pi_{i}^{a}(x)=\left[\begin{array}{cc}
\overline{\mathbf{G}} & a d_{g_{0}} \overline{\mathbf{G}} \\
d\left(L_{g_{0}}^{\gamma_{i}+1} h_{i}\right) \overline{\mathbf{G}} & d\left(L_{g_{0}}^{\gamma_{i}+1} h_{i}\right) a d_{g_{0}} \overline{\mathbf{G}} \\
d\left(L_{g_{0}}^{\gamma_{i}} h_{i}\right) \overline{\mathbf{G}} & d\left(L_{g_{0}}^{\gamma_{i}} h_{i}\right) a d_{g_{0}} \overline{\mathbf{G}} \\
0 & d\left(L_{g_{0}}^{\gamma_{i}} h_{i}\right) \overline{\mathbf{G}}
\end{array}\right]
\end{aligned}
$$

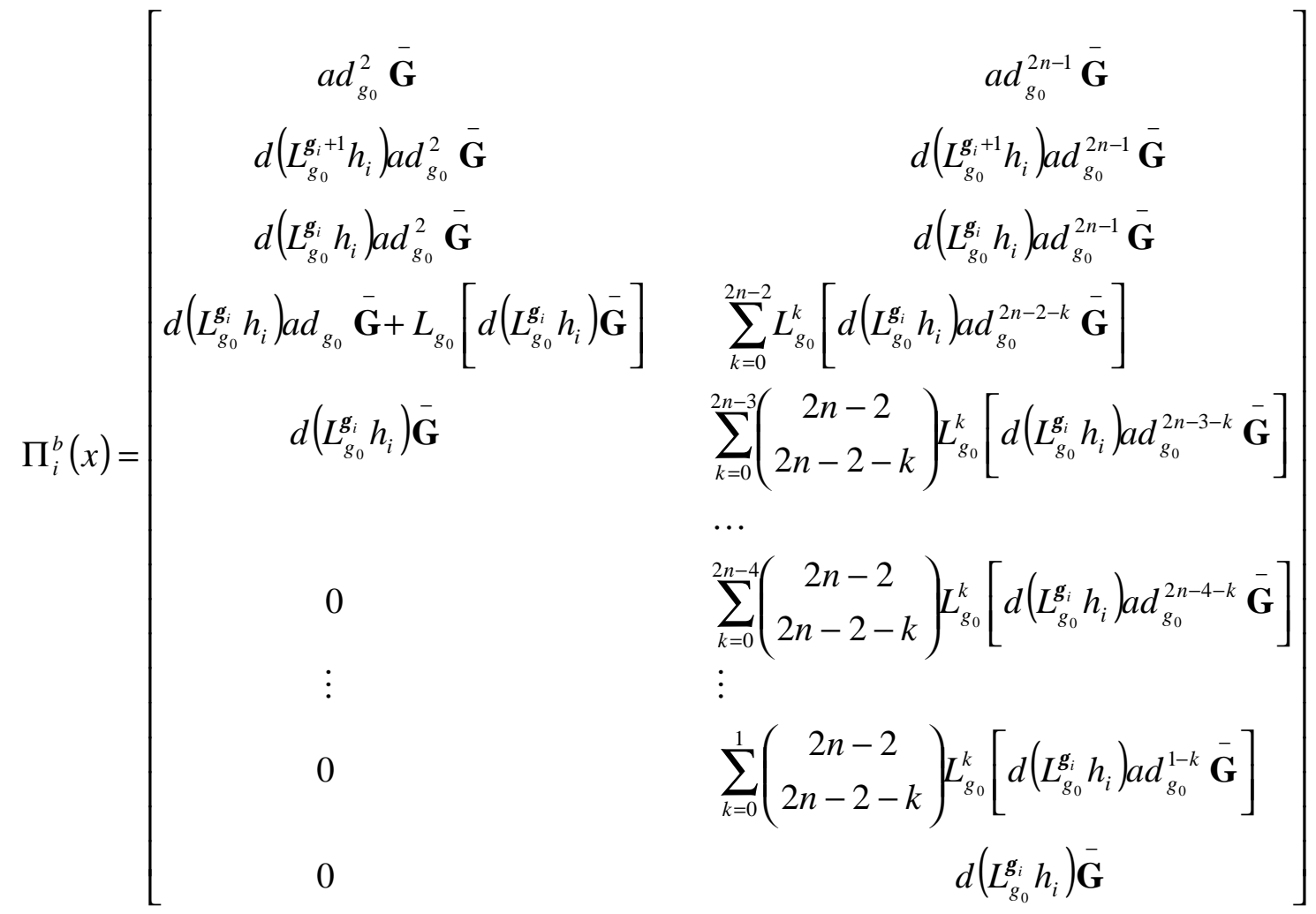

The design equations (3.10) play a fundamental role in our approach, since on the basis of these equations we will derive the necessary and sufficient conditions for the i/o linearization with simultaneous decoupling problem via restricted state feedback. Furthermore, solving the Design Equations for $\left\{a_{i}(x), \mathbf{B}(\mathbf{x})\right\}$ we can determine a solution for the restricted feedback control law.

Remark 3.1. Clearly, if the Design Equations (3.10) are solved for $\left\{a_{i}(x), \mathbf{B}(\mathbf{x})\right\}$ for some $\rho_{k, i}, i \in J_{m}$ and $k \in\{0\} \cup J_{2 n}$, then the Markov parameters of the i/o linearized and decoupled closed-loop can be determined. That is, the design equations (3.10) provide not only the pair $\left\{a_{i}(x), \mathbf{B}(\mathbf{x})\right\}$ of the control law but also the morphological characteristics of the closed-loop system. 


\section{Necessary and sufficient conditions}

In this Section we will establish the necessary and sufficient conditions for the solvability of the $\mathrm{i} / \mathrm{o}$ linearization with simultaneous decoupling problem using restricted state feedback. To this end, define the vector $\delta^{*}(x)$ by

$$
\delta^{*}(x) \stackrel{\Delta}{=}\left[L_{g_{0}}^{\gamma_{1}} h_{1}(x) \ldots L_{g_{0}}^{\gamma_{p}} h_{p}(x)\right]^{T}
$$

Based on the definition of $\delta^{*}(x)$, equation (3.10a), for $i \in J_{p}$, may be expressed in a compact matrix form as follows

where

$$
d\left(\delta^{*}(x)\right) \mathbf{G}(\mathbf{x}) \mathbf{B}(\mathbf{x})=\mathbf{P}_{\mathbf{0}}
$$

$$
\mathbf{P}_{\mathbf{0}} \stackrel{\Delta}{=} \underset{i \in J_{p}}{\operatorname{diag}}\left\{\boldsymbol{\rho}_{0, i}^{-1}\right\}
$$

and where use was made of the definition of $\overline{\mathbf{G}}(x)$ and of the fact that $\rho_{0, i} \neq 0, i \in J_{p}$. Next, consider the matrices $\Gamma_{0}(x)$ and $\Gamma_{1}(x)$ defined as

$$
\begin{aligned}
& \Gamma_{0}(x)=\left[\begin{array}{c}
d\left(h_{1}(\mathbf{x})\right) \mathbf{G}(\mathbf{x}) \\
\vdots \\
d\left(L_{g_{0}}^{\gamma_{1}-1} h_{1}(x)\right) \mathbf{G}(\mathbf{x}) \\
\ldots \ldots \ldots \ldots \ldots \ldots \ldots \ldots \ldots \ldots \ldots \ldots \ldots \ldots \\
\vdots \\
\ldots \ldots \ldots \ldots \ldots \\
d\left(h_{p}(x)\right) \mathbf{G}(\mathbf{x}) \\
\vdots \\
d\left(L_{g_{0}}^{\gamma_{p}-1} h_{p}(x)\right) \mathbf{G}(\mathbf{x})
\end{array}\right] \\
& \Gamma_{1}(x) \stackrel{\Delta}{=}\left[\frac{\Gamma_{0}(x)}{d\left(\delta^{*}(x)\right) \mathbf{G}(\mathbf{x})}\right]
\end{aligned}
$$

The following Theorem can now be formulated.

\section{Theorem 4.1.}

The i/o linearization with simultaneous decoupling problem under the restricted state feedback law (2.2), is solvable if and only if, there exists a set of nonnegative integers $\left\{\gamma_{1}, \ldots, \gamma_{p}\right\}$ such that the following condition holds

$$
\operatorname{rank}\left\{\Gamma_{1}(x)\right\}-\operatorname{rank}\left\{\Gamma_{0}(x)\right\}=p, \forall x \text { around } \mathbf{x}_{\mathbf{0}}
$$

Proof(Necessity). From the Definition 2.2 of the characteristic numbers $\gamma_{i}{ }^{\prime} s$ we can easily obtain that

$$
\Gamma_{0}(\mathbf{x}) \mathbf{B}(\mathbf{x})=\mathbf{0}
$$

Combining (4.5) with (4.1) and using definition (4.3) we can easily take

$$
\Gamma_{1}(\mathbf{x}) \mathbf{B}(\mathbf{x})=\left[\frac{\mathbf{0}}{\mathbf{P}_{\mathbf{0}}}\right]
$$

It is obvious that if (4.6) has a solution for $\mathbf{B}(\mathbf{x})$, then the following condition holds 


$$
\operatorname{rank}\left\{\Gamma_{1}(x)\right\}=\operatorname{rank}\left\{\left[\Gamma_{1}(x): \frac{0}{P_{0}}\right]\right\}
$$

for any $\mathbf{x}$ around $\mathbf{x}_{\mathbf{0}}$. Moreover, since $\mathbf{P}_{\mathbf{0}}$ is invertible, by construction, it also holds that

$$
\operatorname{rank}\left\{\left[\Gamma_{1}(x): \frac{0}{P_{0}}\right]\right\}=\operatorname{rank}\left\{\left[\frac{\Gamma_{0}(x)}{d\left(\delta^{*}(x)\right) \mathbf{G}(\mathbf{x})}: \frac{0}{P_{0}}\right]\right\}=\operatorname{rank}\left\{\Gamma_{0}(x)\right\}+p
$$

for any $\mathbf{x}$ around $\mathbf{x}_{\mathbf{0}}$, where use was made of the definitions (4.3). Combining (4.8) with (4.7), we finally arrive at (4.4).

(Sufficiency). Assume that there exists a set of integers $\left\{\gamma_{1, s}, \ldots, \gamma_{p, s}\right\}$ which satisfies (4.4). Then, (4.6) is solvable for $\mathbf{B}(\mathbf{x})$ and hence (4.1) is solvable with respect to $\mathbf{B}(\mathbf{x})$ for any diagonal and invertible real matrix $\mathbf{P}_{\mathbf{0}}$. Denote by $\hat{\mathbf{B}}(\mathbf{x})$, the solution of (4.6) with respect to $\mathbf{B}(\mathbf{x})$, for some

$$
\mathbf{P}_{\mathbf{0}}=\hat{\mathbf{P}}_{\mathbf{0}}=\underset{i \in J_{p}}{\operatorname{diag}}\left\{\begin{array}{cc}
\hat{\rho}^{-1} & -1
\end{array}\right\}
$$

where $\hat{\rho}_{0, i} \neq 0$. In this case,(3.10b) is always solvable with respect to $a_{i}(x), \rho_{k, i}, k \in\left\{0, J_{2 n}\right\}$ and $i \in J_{p}$.Indeed, $\hat{a}_{i}(x), \rho_{k, i}, k \in\left\{0, J_{2 n}\right\}$ and $i \in J_{p}$,defined by

$$
\begin{gathered}
\hat{a_{i}}(x) \stackrel{\Delta}{=}-\hat{\rho}_{o, i} L_{g_{0}}^{\gamma_{i, s}+1} h_{i}(x) \\
\hat{\rho_{0, i}} \neq 0, \hat{\rho}_{k, i}=0, k \in J_{2 n}
\end{gathered}
$$

is a solution of (3.10b) with respect to $a_{i}(x), \rho_{k, i}, k \in\{0\} \cup J_{2 n}$ and $i \in J_{p}$, respectively. This completes the proof of the present Theorem.

From the proof of Theorem 4.1, it becomes clear that an admissible solution for the pair $\{\mathbf{a}(\mathbf{x}), \mathbf{B}(\mathbf{x})\}$ of the control law (2.2) can be determined by (4.9a) and by solving equation (4.6) with respect to $\mathbf{B}(\mathbf{x})$. To find a solution of (4.6) with respect to $\mathbf{B}(\mathbf{x})$, let $\hat{\Gamma}_{0}(x), \delta^{*}(x)$ and $\hat{\Gamma}_{1}(x)$ be the matrices defined as in (4.3) for some integers $\left\{\gamma_{1, s}, \ldots, \gamma_{p, s}\right\}$ which satisfy (4.4). Then equation (4.6) may be rewritten as

$$
\begin{gathered}
\hat{\Gamma}_{0}(x) \hat{B}(x)=0 \\
d\left(\hat{\delta}^{*}(x)\right) \hat{\mathbf{G}}(\mathbf{x}) \hat{\mathbf{B}}(\mathbf{x})=\hat{\mathbf{P}}_{\mathbf{0}}
\end{gathered}
$$

where $\hat{\mathbf{P}}_{\mathbf{0}}$ is defined as in the sufficient part of the proof of Theorem 3.2. Furthermore, denote by $\sigma$ the integer

$$
\sigma=\left\{\sum_{i=1}^{p} \gamma_{i, s}-\operatorname{rank}\left[\Gamma_{0}(x)\right]\right\}, \forall x \text { around } \mathbf{x}_{\mathbf{0}}
$$

and by $\hat{\mathbf{w}}(\mathbf{x})$ the mx $\sigma$ full column rank (around $\mathbf{x}_{\mathbf{0}}$ ) matrix which is orthogonal to $\hat{\Gamma_{0}}(x)$. Then, the solution of (4.10a) has the form 


$$
\hat{\mathbf{B}}(\mathbf{x})=\hat{\mathbf{W}}(\mathbf{x}) \hat{\Lambda}(\mathbf{x})
$$

where $\hat{\Lambda}(\mathbf{x})$ is an arbitrary $\sigma \mathrm{xp}$ matrix valued function of $\mathbf{x}$. Introducing (4.11) into (4.10b) the later may be reduced to

$$
\hat{\mathbf{R}}(\mathbf{x}) \hat{\mathbf{M}}(x)=\hat{\mathbf{P}}_{0}
$$

where

$$
\begin{gathered}
\hat{\mathbf{R}}(\mathbf{x})=d\left(\hat{\boldsymbol{\delta}}^{*}(x) \hat{\mathbf{G}}(\mathbf{x})\right. \\
\hat{\mathbf{M}}(\mathbf{x})=\hat{\mathbf{W}}(\mathbf{x}) \hat{\Lambda}(\mathbf{x})
\end{gathered}
$$

Observe now that

$$
\operatorname{rank}[\hat{\mathbf{R}}(\mathbf{x})]=\mathrm{p}, \forall x \text { around } \mathbf{x}_{\mathbf{0}}
$$

Then the solution of (4.12) with respect to $\hat{\mathbf{M}}(\mathbf{x})$ will be given by

$$
\hat{\mathbf{M}}(\mathbf{x})=\hat{\mathbf{R}}^{T}(\mathbf{x})\left[\hat{\mathbf{R}}(\mathbf{x}) \hat{\mathbf{R}}^{T}(\mathbf{x})\right]^{-1} \hat{\mathbf{P}}_{0}
$$

Since $\hat{\mathbf{W}}(\mathbf{x})$ is injective by construction, equation (4.13b) has a unique solution with respect to $\hat{\Lambda}(\mathbf{x})$. This solution has the form

$$
\hat{\Lambda}(\mathbf{x})=\left[\hat{\mathbf{W}}^{T}(\mathbf{x}) \hat{\mathbf{W}}(\mathbf{x})\right]^{-1} \hat{\mathbf{W}}^{T}(\mathbf{x}) \hat{\mathbf{M}}(\mathbf{x})
$$

Finally, introducing expressions (4.14) and (4.15) into (4.11), the matrix valued function $\hat{\mathbf{B}}(\mathbf{x})$ will be given by

$$
\hat{\mathbf{B}}(\mathbf{x})=\hat{\mathbf{W}}(\mathbf{x})\left[\hat{\mathbf{W}}^{T}(\mathbf{x}) \hat{\mathbf{W}}(\mathbf{x})\right]^{-1} \hat{\mathbf{W}}^{T}(\mathbf{x}) \mathbf{x} \hat{\mathbf{R}}^{T}(\mathbf{x})\left[\hat{\mathbf{R}}(\mathbf{x}) \hat{\mathbf{R}}^{T}(\mathbf{x})\right]^{-1} \hat{\mathbf{P}_{0}}
$$

With respect to the vector valued function $\mathbf{a}(\mathbf{x})$ of the control law (2.2), an admissible solution will be given by

$$
\hat{\mathbf{a}}(\mathbf{x})=-\hat{\mathbf{P}}_{0}^{-1} \hat{\mathbf{a}}^{*}(\mathbf{x})
$$

where $\hat{\mathbf{a}}^{*}(\mathbf{x}) \stackrel{\Delta}{=}\left[L_{g_{0}}^{\gamma_{i, s}+1} h_{1}(x) \ldots L_{g_{0}}^{\gamma_{p, s}+1} h_{p}(x)\right]^{T}$. To derive (4.17), use was made of (4.9a).

The solution for $\hat{\mathbf{a}}(\mathbf{x}), \hat{\mathbf{B}}(\mathbf{x})$ of the control law (2.2) derived above corresponds to $\hat{\rho}_{o, i} \neq 0, \hat{\rho}_{k, i}=0, k \in J_{2 n}$. Hence using relations (3.6), the Markov parameters of the closed-loop system have the form

$$
\hat{\mu}_{o, i} \neq \hat{\rho}_{o, i}^{-1}, \hat{\mu}_{k, i}=0, \text { for } k \in J_{2 n-1}
$$

The i/o description of the i/o linearized and decoupled closed-loop system may be realised as a transfer function of the form

$$
\mathrm{H}(\mathrm{s})=\operatorname{diag}_{i \in J_{p}}\left\{s^{-\left(\gamma_{i, s}+1\right)} \hat{\mu}_{o, i}\right\}
$$


where use was made of (3.4) and (3.5).

\section{Conclusions}

A new approach to the i/o linearization with simultaneous decoupling problem of nonsquare nonlinear systems via restricted static state feedback is presented in this paper. The proposed approach reduces the determination of all the admissible control laws to the solution of an algebraic and a nonhomogeneous first order partial differential systems of equations. On the basis of these systems of equations simple algebraic criteria for the solvability of the aforementioned problem are established. Moreover, an analytic expression of an admissible restricted static state feedback is derived.

The present approach seems to be more powerful than known techniques, since it provides the fundamental equations needed for the characterisation of all admissible controllers as well as the respective i/o linearized and simultaneously decoupled closed-loop response. It also appears that it can be easily extended to treat other relative problems for nonlinear systems, as for example the disturbance rejection with simultaneous i/o linearization and decoupling problem, via restricted state feedback.

\section{References}

[1]. P.L.Falb and W.A. Wolovich, "Decoupling in the design and synthesis of multivariable control systems", IEEE Trans. Autom. Contr., Vol. AC-12, pp. 651-659, 1967.

[2]. W.A.Porter, "Decoupling of and inverse for time-varying linear systems", IEEE Trans. Autom. Contr., Vol. AC-14, pp.378-380,1967.

[3]. W.A. Porter, "Diagonalization and inverses for nonlinear systems", Int. J. Contr., Vol.10, pp. 252-264, 1970.

[4]. I.J. Ha and E.G. Gilbert, "A complete characterization of decoupling control laws for a general class of nonlinear systems", IEEE Trans. Autom. Contr., Vol. AC-31, pp.823-830, 1986.

[5]. Xiaohua Xia, "Parameterization of decoupling control laws for affine nonlinear systems", IEEE Trans. Autom. Contr., Vol. AC-38, pp.916-928, 1993.

[6]. B.S. Morgan, "The synthesis of linear multivarible systems by state variable feedback", IEEE Trans. Autom. Contr., Vol. AC-9, pp. 405-411, 1964.

[7]. N.Suda and K.Umahashi, "Decoupling of nonsquare systems: A necessary and sufficient condition in terms of infinite zeros", Proc. $9^{\text {th }}$ IFAC World Congress, Budapest, Vol.1, pp. 8893, 1984.

[8]. J.Descusse, J.F.Lafay and M.Malabre, "A syrvey on Morgan's problem", Proc. $25^{\text {th }}$ IEEE C.D.C., Vol.2, pp. 1289-1294, Athens, Greece, 1986.

[9]. C.Comault, J.Descousse, J.M.Dion, J.F. Lafay and M.Malabre, "New decoupling invariants: The essential orders", Int. J. Contr., Vol. 44, pp. 689-700, 1986.

[10]. J.Descusse, J.F. Lafay and M.Malabre, "Solution to Morgan's problem", IEEE Trans. Autom. Contr., Vol. AC-33, pp.732-739, 1988.

[11]. H.A.N. Herrera and J.F.Lafay, "New results about Morgan's problem", IEEE Trans Autom. Contr., Vol.38, pp.1834-1838, 1993.

[12]. A.Glumineau and C.H.Moog, "Nonlinear Morgan's problem: Case of $(\mathrm{p}+1)$ inputs and $\mathrm{p}$ outputs", IEEE Trans Autom. Contr., Vol. AC-37, pp.1067-1072, 1992.

[13]. S.Kamiyama and K.Furuta, "Decoupling by restricted state feedback", IEEE Trans Autom. Contr., Vol.21, pp.413-415, 1976.

[14]. J.Descusse, J.F.Lafay and V.Kucera, "Decoupling by restricted state feedback: The general case", IEEE Trans Autom. Contr., Vol. AC-29, pp.79-81, 1984. 
[15]. K.G. Arvanitis, "Simultaneous uniform disturbance rejection and decoupling of nonsquare linear time varying analytic systems by restricted state feedback", IMA J. Math. Control Infor., in press.

[16]. T.J.Tarn and W.Zhan, "Input-Output decoupling and linearization via restricted static state feedback", proc. $11^{\text {th }}$ IFAC World Congress, Tallin, Vol.3, pp.287-292, 1991.

[17]. A.S. Tsirikos, "New techniques for the analysis and design of linear and nonlinear systems", PhD Thesis, NTUA, Athens, under completion. 